\title{
Surface-Mediated Protein Unfolding as a Search Process for Denaturing Sites
}

\author{
James S. Weltz, Daniel K. Schwartz ${ }^{*}$, and Joel L. Kaar ${ }^{*}$
}

Department of Chemical and Biological Engineering, University of Colorado, Boulder, CO

$$
\text { 80309, USA }
$$

\section{SUPPORTING INFORMATION}

${ }^{*}$ Corresponding Authors:

Joel L. Kaar

University of Colorado Boulder

Department of Chemical and Biological Engineering

Campus Box 596

Boulder, CO 80309

Tel: (303) 492-6031

Fax: (303) 492-4341

Email: joel.kaar@,colorado.edu

Daniel K. Schwartz

University of Colorado Boulder

Department of Chemical and Biological Engineering

Campus Box 596

Boulder, CO 80309

Tel: (303) 735-0240

Fax: (303) 492-4341

Email: daniel.schwartz@,colorado.edu

Supplementary Materials:

1) Supplementary Figures

S1) SDS-PAGE of T4 $\mathrm{L}_{\text {FRET }}$

S2) In-Gel Fluorescence of Labeled T4 $\mathrm{L}_{\mathrm{FRET}}$

S3) Circular Dichroism Spectra of Unlabeled T4L WT* and T4 $\mathrm{L}_{\text {FRET }}$

S4) Ensemble Denaturation of Labeled T4 $\mathrm{L}_{\mathrm{FRET}}$

S5) Histogram of $d$-values from Single-Molecule Analysis of T4 $\mathrm{L}_{\mathrm{FRET}}$ Unfolding on FS

S6) Frames to Unfolding of T4 $\mathrm{L}_{\mathrm{FRET}}$ Upon Re-adsorption on FS

S7) Surface Coverage vs Solution Concentration of T4L WT*

S8) Histogram of the Number of Adsorption Events for T4L WT* on FS 


\section{Supplementary Figures:}

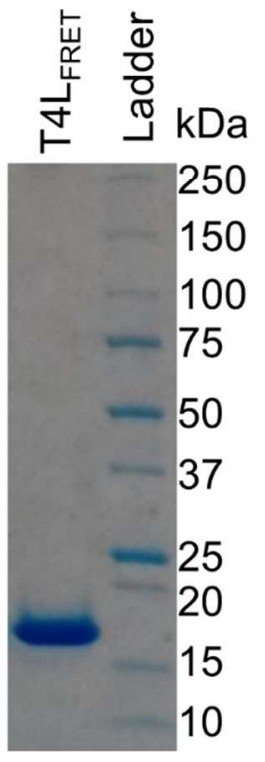

Figure S1. Coomassie-stained SDS-PAGE of T4LFRE after purification. The gel contains a single band of approximately $18 \mathrm{kDa}$ that corresponds to the expected molecular weight of T4L $L_{\text {FRET }}$. 


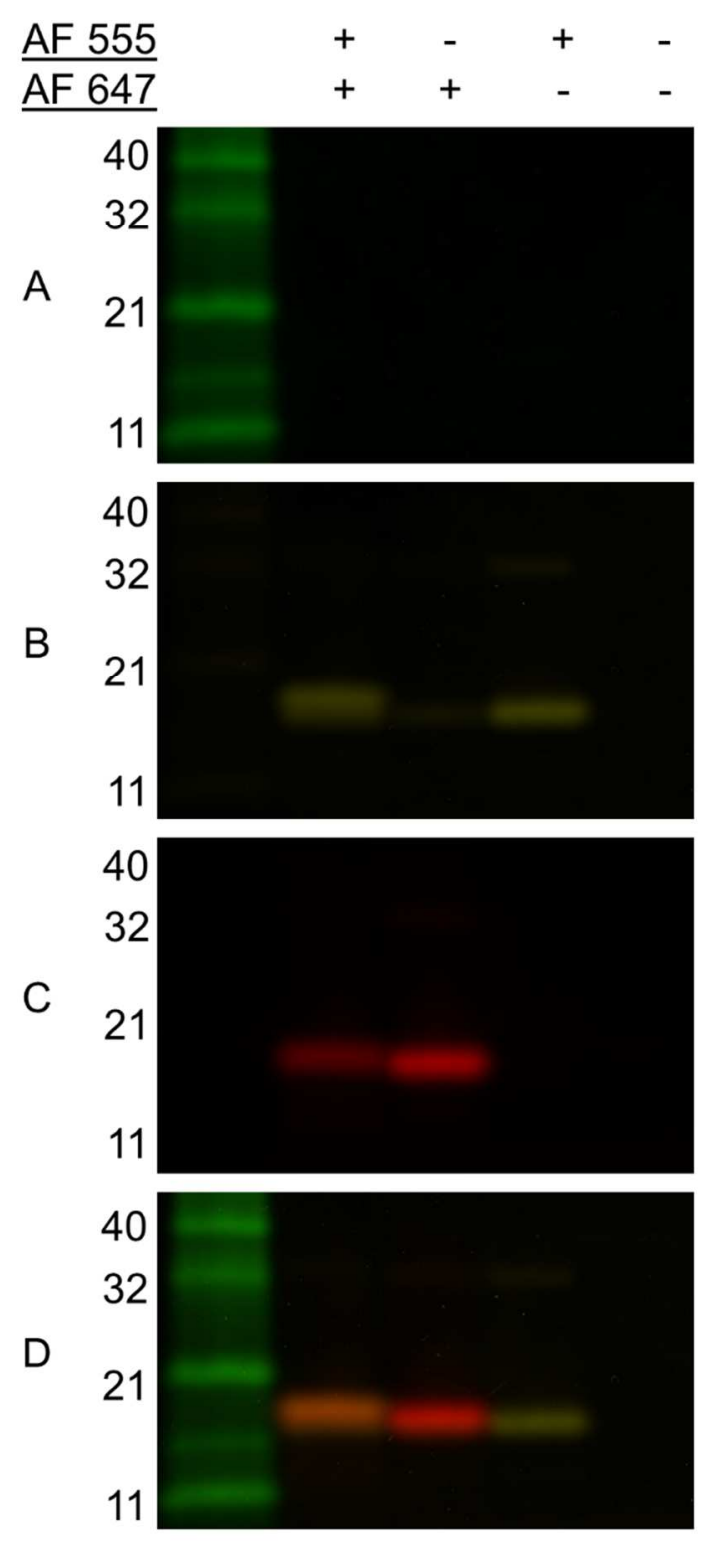

Figure S2. In-gel fluorescence imaging of labeled T4 $\mathrm{L}_{\mathrm{FRET}}$ with direct excitation of (A) Alexa 488 (for the protein ladder), (B) Alexa 555, (C) Alexa 647, and (D) the composite (i.e., overlay) image confirmed covalent attachment of donor and acceptor dye molecules. 


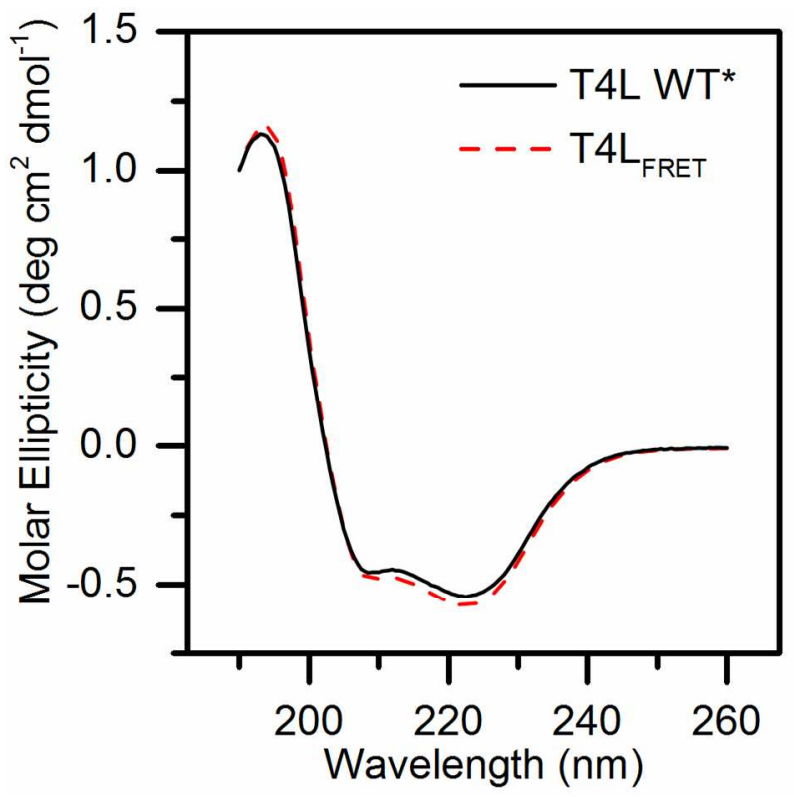

Figure S3. Circular dichroism spectra (Chirascan Plus, Applied Photophysics) for unlabeled T4L $\mathrm{WT}^{*}$ and T4L $\mathrm{L}_{\mathrm{FRET}}$ was measured in $50 \mathrm{mM}$ sodium phosphate, $\mathrm{pH} 6.80$ at $1 \mathrm{mg} / \mathrm{mL}$ protein concentration. The spectra represent the average of five successive scans from 190-260 nm in 0.5 $\mathrm{nm}$ increments with an integration time of $0.5 \mathrm{~s}$ per increment. 


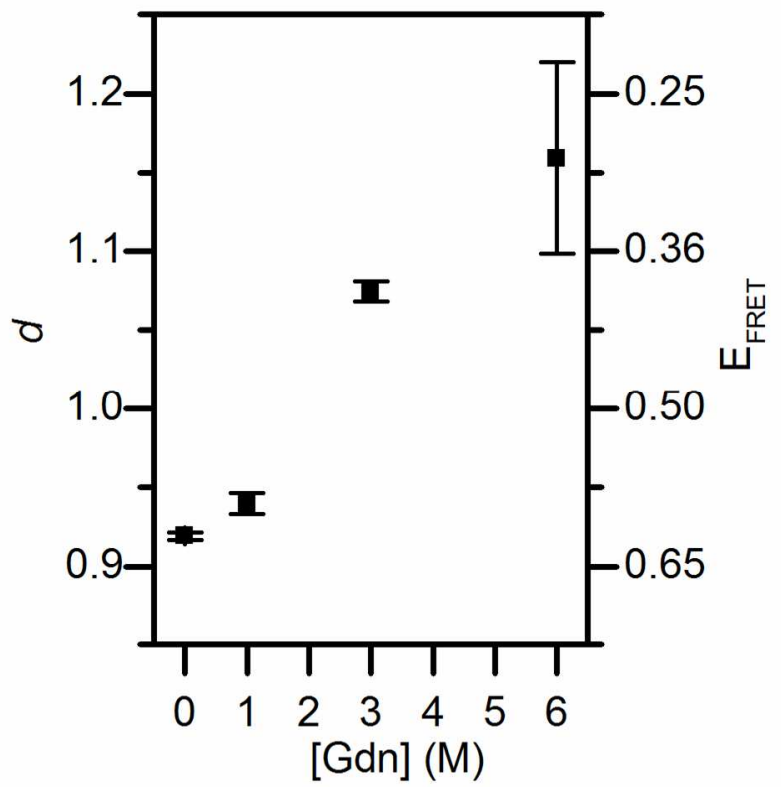

Figure S4. Ensemble denaturation of labeled T4 $\mathrm{L}_{\mathrm{FRET}}$ in solution, showing a monotonic increase in $d$-value and corresponding decrease in FRET efficiency with increasing denaturant. Solutions containing $5 \mu \mathrm{g} / \mathrm{mL}$ of labeled T4LFRET were incubated at room temperature in $50 \mathrm{mM}$ sodium phosphate buffer, $\mathrm{pH}$ 6.80, containing 0-6 M guanidine hydrochloride (Gdn) for $1 \mathrm{~h}$. FRET was measured by exciting the protein solution at $555 \mathrm{~nm}$ and measuring fluorescence emission of the donor and acceptor at 580 and $665 \mathrm{~nm}$, respectively, using a Fluoromax-4 (Horiba) fluorimeter. Error bars represent the standard deviation of $d$-values obtained from three independent replicates. The larger inter-replicate error at the highest denaturant concentration (6 M Gdn) may be due to a number of plausible explanations, including formation of soluble aggregates, low acceptor intensity, and inherent conformational heterogeneity of the unfolded state. 


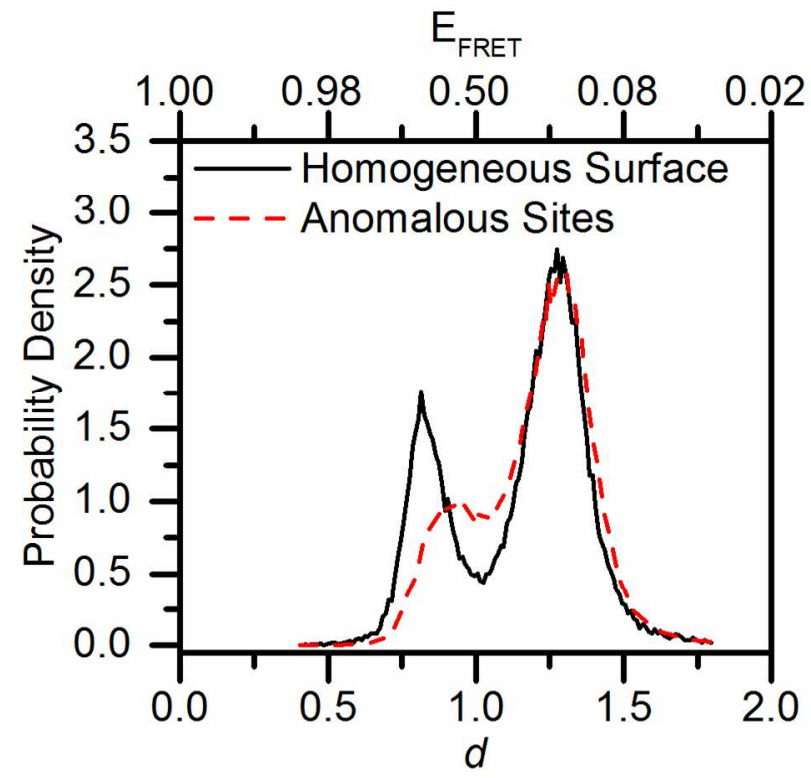

Figure S5. Distribution of $d$-values for T4 $\mathrm{L}_{\mathrm{FRET}}$ for approximately $10^{6}$ observations on FS, which was used to identify molecular signatures of folded and unfolded states. Each observation was segmented based on the location as either on the homogeneous surface or regions with anomalously high numbers of adsorption events. Histograms of $d$-values for the homogeneous surface showed two well-separated populations centered at $d=0.85$ and $d=1.27$, corresponding to folded and unfolded states, respectively. The threshold $d$-value of 1.05 to classify molecules as folded or unfolded corresponds to the minimum probability between the two observed populations. Compared to on the homogenous surface, a greater fraction of protein molecules adsorbed to anomalous sites were unfolded, suggesting that the anomalous sites were denaturing. 


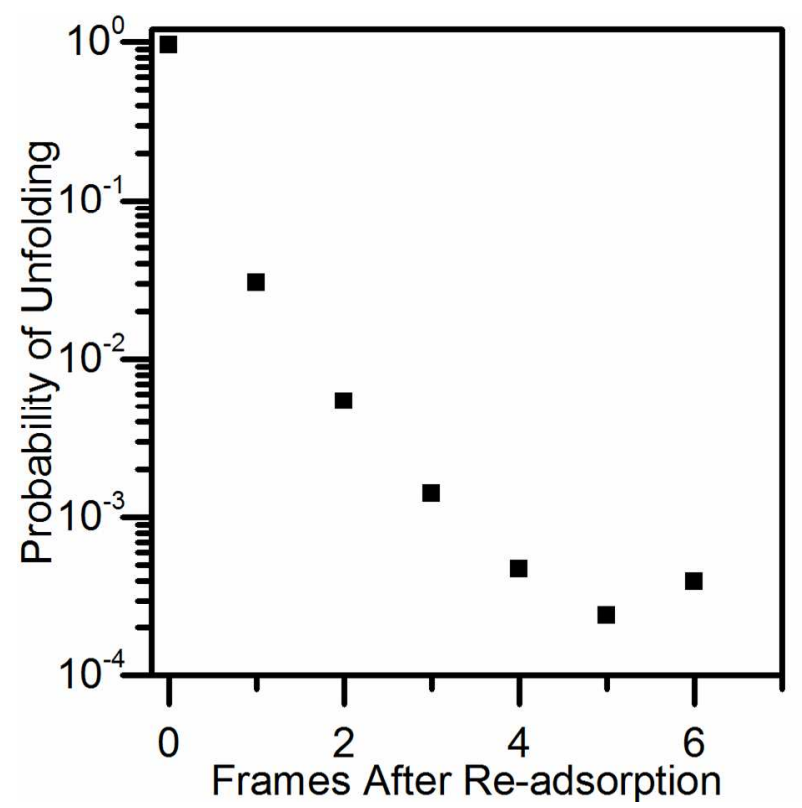

Figure S6. The distribution of the number of frames in which a folded protein molecule appears immobile before unfolding. Motion was considered significant if the protein exhibited an apparent displacement $>50 \mathrm{~nm}$ between frames. 


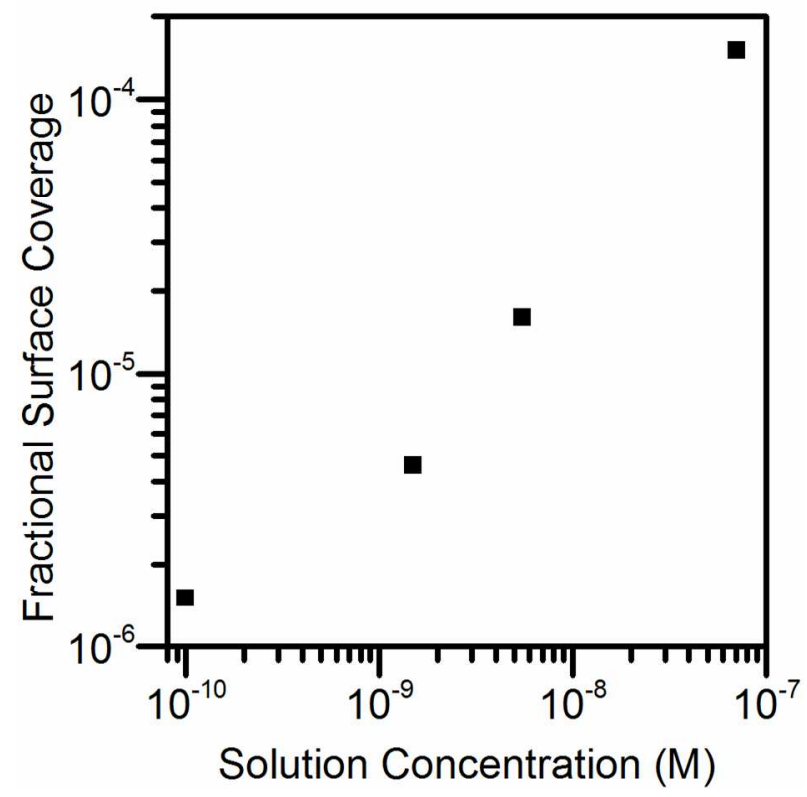

Figure S7. Fractional surface coverage of unlabeled T4L WT* as a function of the concentration of T4L WT* in the bulk solution exposed to FS. Surface coverages were estimated from the ratio of labeled-to-unlabeled protein used for each condition, the number of labeled molecules adsorbed, and the estimated footprint of T4L WT* from the crystal structure (PDB 1L63). The footprint of T4L WT* was estimated assuming a "side-on" orientation and rectangular projection with a width $(3 \mathrm{~nm})$ and length $(4 \mathrm{~nm})$ using the maximum dimensions measured in the crystal structure (PDB 1L63) as mentioned in the main paper. 

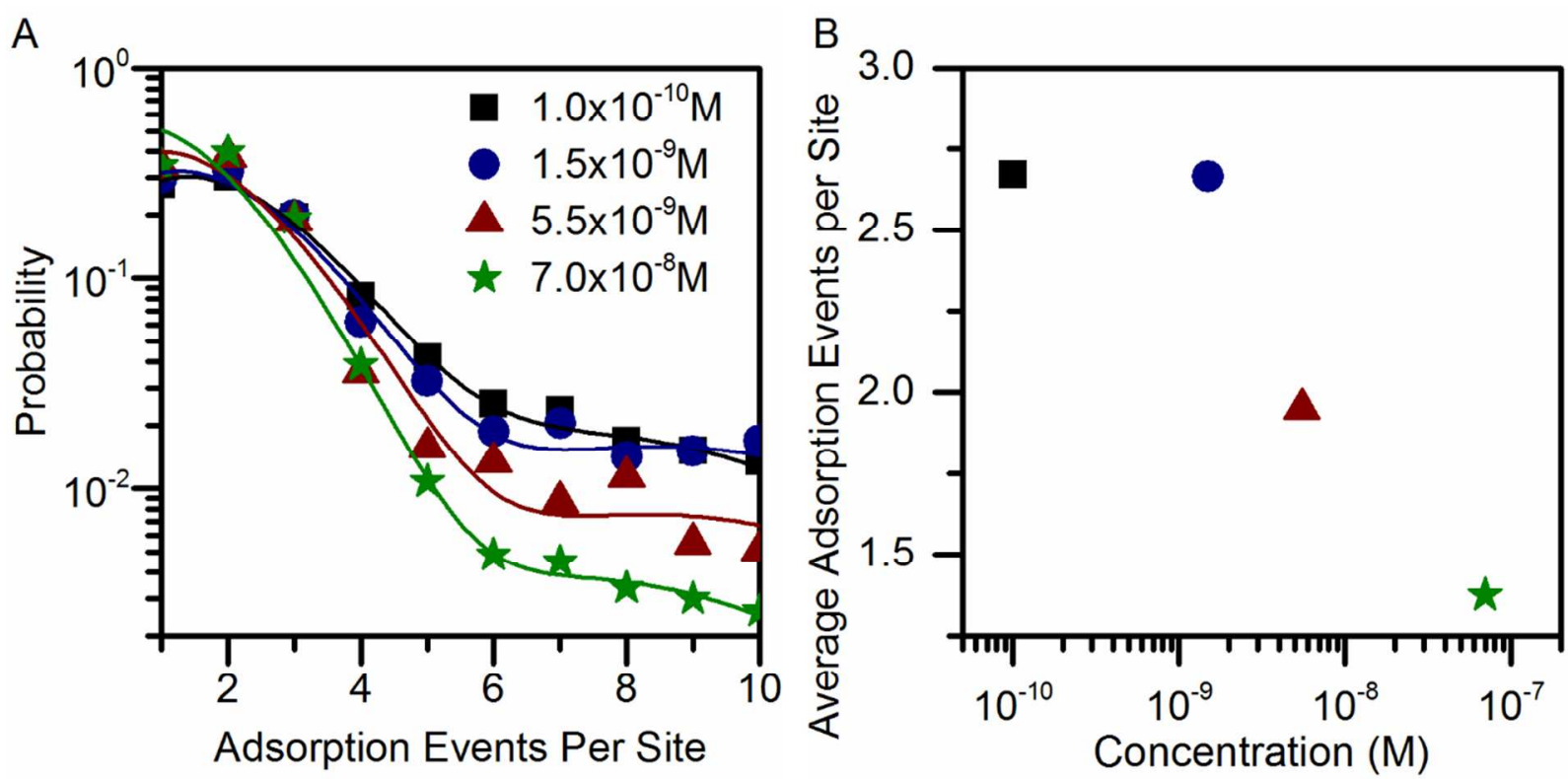

Figure S8. (A) Sites that demonstrated anomalously high numbers of adsorption events were identified and the number of adsorption events to each continuous site was quantified. The adsorption site histogram was fit to a three-component Poisson mixture model. (B) The average number of adsorption events per site calculated from the fitted Poisson mixture model. 